\title{
Dynamical topological excitations in parafermion chains
}

\author{
Vilja Kaskela and J. L. Lado ${ }^{0}$ \\ Department of Applied Physics, Aalto University, 00076 Aalto, Espoo, Finland
}

(Received 14 November 2020; accepted 11 January 2021; published 29 January 2021)

\begin{abstract}
Parafermions are elusive fractional excitations potentially emerging in fractional quantum Hallsuperconductor junctions and represent one of the major milestones in fractional quantum matter. However, generic models of parafermions are not analytically solvable, and understanding their topological modes is a bigger challenge than conventional Majorana modes. Here, by using a combination of tensor network and kernel polynomial techniques, we demonstrate the emergence of zero modes and finite energy excitations in many-body parafermion chains. We show the appearance of zero-energy modes in the many-body spectral function at the edge of a topological parafermion chain, their relation with the topological degeneracy of the system, and we compare their physics with the Majorana bound states of topological superconductors. We demonstrate the robustness of parafermion topological modes with respect to a variety of perturbations, and we show how weakly coupled parafermion chains give rise to in-gap excitations. Our results exemplify the versatility of tensor network methods for studying dynamical excitations of interacting parafermion chains and highlight the robustness of topological modes in parafermion models.
\end{abstract}

DOI: 10.1103/PhysRevResearch.3.013095

\section{INTRODUCTION}

Unconventional excitations in quantum materials are a central research area in modern condensed matter physics [1,2]. Paradigmatic examples of unconventional excitations are the edge modes of topological insulators $[1,3,4]$, including quantum anomalous Hall insulators and quantum spin Hall insulators [5]. Solely, these systems have attracted a great amount of attention for their potential for dissipationless electronics and spintronics. Topological superconductors [6,7] represent another instance in which topological excitations have a major role. In particular, the emergence of Majorana zero modes $[7,8]$ in these systems puts forward the possibility of using superconductors as a noise-resilient platform for topological quantum computing [9-11].

Interest in topological superconductors started with the first proposals to realize artificial $p$-wave superconducting in a variety of platforms [12-21], by combining strongspin orbit coupling effects, superconducting proximity effect and exchange fields. Majorana bound states can be generalized to a wider class of topological excitations, known as parafermions [22]. In particular, parafermions realize quantum excitations with generalized commutation relations, providing a powerful platform for topological quantum computing, overcoming a limitation of Majorana bound states $[9,10,23]$. In contrast to fermions, parafermions do not exist in nature, and thus they must be artificially engineered.

Published by the American Physical Society under the terms of the Creative Commons Attribution 4.0 International license. Further distribution of this work must maintain attribution to the author(s) and the published article's title, journal citation, and DOI.
Following the previous point, a variety of proposals involving fractional quantum Hall states with superconductivity have been put forward for their artificial engineering [24-36], inspired by the success of proposals for Majorana bound states.

Majorana bound states can be described in an effectively single-particle picture with the Bogoliuvov-de-Gennes formalism. The robustness of these modes to perturbations stems from their topological origin, which is associated to the existence of a single-particle nontrivial topological invariants. Interestingly, the inclusion of many-body interactions turns those topological models much richer [37]. Ultimately, parafermion models represent a much bigger challenge from the theoretical point of view. This stems from the fact that parafermion models cannot be solved analytically in general [38,39], and no general proof exists to demonstrate ground state degeneracies. As a result, models for parafermions become full-fledged many-body problems, requiring a full many-body treatment. Parafermion models are substantially less explored than their Majorana counterparts. In particular, the computation of dynamical excitations in parafermion chains remains a challenging problem due to the genuine many-body nature of the problem, and the lack of exact analytical tools for its generic treatment.

Here, using a combination of tensor network and kernel polynomial techniques, we show the emergence of edge and interface topological excitations in parafermion chains. In particular, here we demonstrate that parafermion chains show edge zero modes that are resilient to a variety of parafermion many-body interactions, and that weak coupling between parafermion zero modes give rise to in-gap excitations at finite energy at the interface (Fig. 1). Furthermore, we compare the phenomenology of these parafermion chains with those of Majorana excitations in topological superconductors. Our manuscript is organized as follows. First, in Sec. II, we present 
(a)

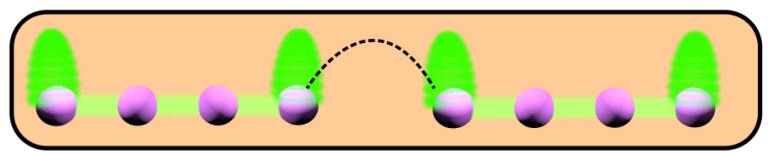

(b)

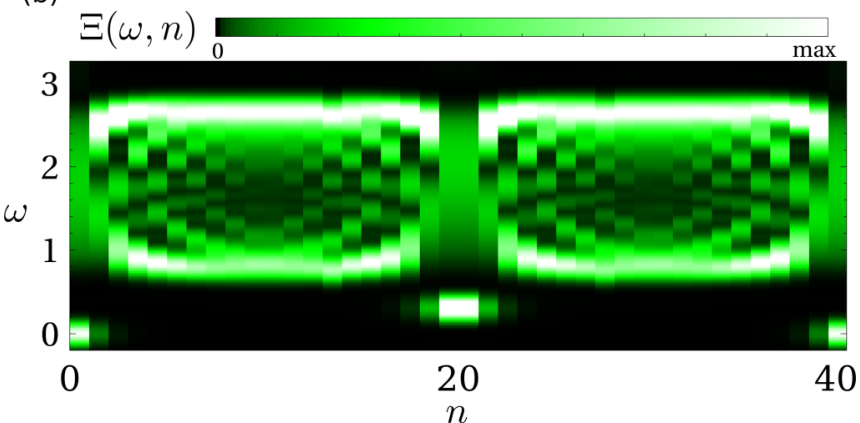

(c)

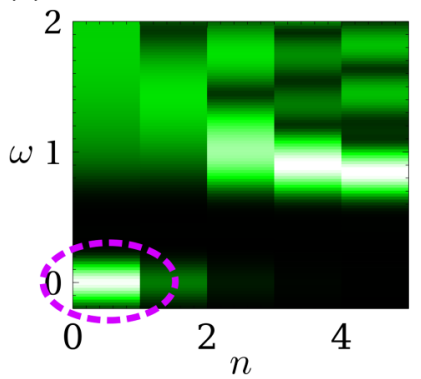

(d)

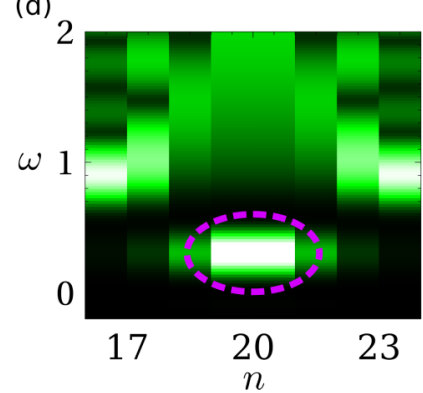

FIG. 1. Sketch of two weakly coupled parafermion chains (a), showing the emergence of decoupled topological excitations at the edge and coupled modes at the interface. (b) shows the dynamical spectral function at each site of the chain, showing the emergence of topological zero modes at the edges (c) and finite energy in-gap excitations at the interface (d). Bound states in (c) and (d) are highlighted by the purple dashed circles.

a generalized parafermion model, that simultaneously captures conventional fermions and parafermions, together with a quantum many-body procedure used to solve the system. In Sec. III, we use this formalism to study the dynamical topological modes in a Majorana chain, including the effects of many-body interactions, decoupling and disorder. In Sec. IV, we show that a parafermion chain show emergent zero modes in the spectral function. In Sec. V, we show that perturbations to the parafermion Hamiltonian leave the zero-edge excitation unaffected. In Sec. VI, we show how interfacial in-gap excitations at finite energy emerge at the interface between two parafermion chains. Finally, in Sec. VII, we summarize our conclusions.

\section{MODEL}

\section{A. Clock model and parafermions}

In the following, we will study a one-dimensional model of parafermions [22,36,40-52] exhibiting topological zero modes. Parafermions are generalizations of conventional fermions with $Z_{N}$ symmetry showing generalized commutation relations. Parafermion models are conveniently written from a so-called clock model, involving operators $\tau$ and $\sigma$. The clock operators $\tau$ and $\sigma$ generalize the Pauli $x$ and $z$

matrices, with the following properties:

$$
\sigma^{n}=\tau^{n}=1, \quad \sigma^{\dagger}=\sigma^{n-1}, \quad \tau^{\dagger}=\tau^{n-1},
$$

The integer $N$ determines the type of $Z_{N}$ parafermion considered. In particular, for $N=2$, the conventional algebra of Pauli matrices is recovered, yielding $\sigma^{2}=1$. In contrast, for $N=3$, one recovers the same state upon applying the operator three times. The clock operators allow generalizing the notion of fermions, by promoting the typical Jordan-Wigner algebra to $Z_{N}$ symmetry. The clock operators follow a generalized commutation relation of the form

$$
\sigma \tau=z \tau \sigma
$$

with $z=e^{2 \pi i / n}$. In a parafermion chain, each site is taken to have its own set of parafermion operators $\tau_{j}$ and $\sigma_{j}$. With those local clock operators, the operators for parafermions are derived from the clock operators as $[22,53,54]$

$$
\begin{gathered}
\chi_{j}=\left(\prod_{k=1}^{j-1} \tau_{k}\right) \sigma_{j}, \\
\psi_{j}=\left(\prod_{k=1}^{j-1} \tau_{k}\right) \sigma_{j} \tau_{j},
\end{gathered}
$$

where $\psi$ and $\chi$ correspond to the two parafermion operators. The previous transformation can be understood as a generalized Jordan-Wigner transformation between conventional spin operators and fermionic operators [22]. The Hamiltonian of the parafermion chain is constructed with $\psi_{j}, \chi_{j}, \psi_{j}^{\dagger}$, and $\chi_{j}^{\dagger}$. The parafermionic commutation relation follows from the commutation relations of the clock operators and is given by

$$
\chi_{j} \psi_{k}=z \psi_{k} \chi_{j}, \quad \chi_{j} \chi_{k}=z \chi_{k} \chi_{j}, \quad \psi_{j} \psi_{k}=z \psi_{k} \psi_{j},
$$

for $j<k$. These commutation relations are responsible for the exotic quantum statistics of the chain. In particular, taking $n=2$ recovers the commutation relations for fermions. Given the previous operators, a many-body Hamiltonian for the parafermion chain can be written as

$$
\mathcal{H}=\text { if } \sum_{n} \chi_{n}^{\dagger} \psi_{n}+i \theta \sum_{n} \psi_{n}^{\dagger} \chi_{n+1}+\text { H.c. }
$$

where $f$ is an on-site coupling between parafermions on the same site, and $\theta$ a coupling between parafermions in different sites. We focus on the Hamiltonian of Eq. (6) for simplicity, yet of course more complex Hamiltonians involving parafermion operators can be written. The previous Hamiltonian is known to have a rich phase diagram for complex values of $f$ and $\theta[45,55,56]$, which in particular hosts a phase with many-body topological order. We note that the topological order we study exists only in parafermionic model but not in the clock model. In particular, in the clock chain, the degenerate ground states are not robust to local perturbations. Here we will focus on this topological phase, which is obtained in particular by taking $\theta=1$ and $f=0.5$. In particular, we will be interested in studying the dynamical excitations of the system, which follows from computing the following dynamical correlator:

$$
\Xi(\omega, n)=\left\langle\mathrm{GS}\left|\chi_{n}^{\dagger} \delta\left(\omega-\mathcal{H}+E_{\mathrm{GS}}\right) \psi_{n}\right| \mathrm{GS}\right\rangle,
$$


where $|\mathrm{GS}\rangle$ is the many-body ground state of the system and $E_{\mathrm{GS}}$ the ground-state energy. Due to the genuine many-body nature of this model, we will compute this dynamical correlator numerically using the kernel polynomial tensor network as elaborated in the next section.

\section{B. Kernel polynomial tensor network formalism}

Due to the many-body nature of the Hamiltonian (6), a generic analytic solution cannot, in general, be obtained. To tackle this problem, we will here employ the tensor network formalism [57-62], which is in particular well suited for generic interacting one dimensional problems. In order to compute the dynamical correlators we will use the tensor network kernel polynomial formalism [63-68]. The kernel polynomial method [63] (KPM) allows for the computation of spectral functions directly in frequency space by performing expansion in terms of the Chebyshev polynomials of Eq. (7). For simplicity, we focus our discussion on the rescaled Hamiltonian $\mathcal{H} \rightarrow \overline{\mathcal{H}}$, whose ground state energy is located at $E=0$ and whose excited states are restricted to the interval $[0,1),{ }^{1}$ which can be generically obtained by shifting and rescaling the original Hamiltonian $\mathcal{H}$. The dynamical correlator $\Xi$ for the original Hamiltonian $\mathcal{H}$ can then be recovered by rescaling back the energies in the dynamical correlator $\bar{\Xi}$ of the scaled Hamiltonian $\overline{\mathcal{H}}$. To compute the dynamical correlator $\overline{\boldsymbol{\Xi}}$, we perform an expansion of the form

$$
\bar{\Xi}(\omega)=\frac{1}{\pi \sqrt{1-\omega^{2}}}\left(\mu_{0}+2 \sum_{l=1}^{N_{P}} \mu_{l} T_{l}(\omega)\right),
$$

where $T_{l}$ are Chebyshev polynomials and $N_{P}$ are the number of polynomials considered. The coefficients of the expansion $\mu_{l}$ can be then computed as $\mu_{l}=\left\langle\mathrm{GS}\left|\chi_{n}^{\dagger} T_{l}(\overline{\mathcal{H}}) \psi_{n}\right| \mathrm{GS}\right\rangle$, where $|\mathrm{GS}\rangle$ is computed with the density-matrix renormalization group (DMRG) algorithm [57]. Taking into account the recursion relation of the Chebyshev polynomials $T_{l}(\omega)=$ $2 \omega T_{l-1}(\omega)-T_{l-2}(\omega)$, with $T_{1}(\omega)=\omega$ and $T_{0}(\omega)=1$, the different coefficients $\mu_{l}$ can be computed by iteratively defining the vectors

$$
\begin{gathered}
\left|w_{0}\right\rangle=\psi_{n}|\mathrm{GS}\rangle, \\
\left|w_{1}\right\rangle=\overline{\mathcal{H}}\left|w_{0}\right\rangle, \\
\left|w_{l+1}\right\rangle=2 \overline{\mathcal{H}}\left|w_{l}\right\rangle-\left|w_{l-1}\right\rangle,
\end{gathered}
$$

so that $\left|w_{l}\right\rangle=T_{l}(\overline{\mathcal{H}}) \psi_{n}|\mathrm{GS}\rangle$.

In this way, the coefficients $\mu_{l}$ are computed as $\mu_{l}=$ $\left\langle\mathrm{GS}\left|\chi_{n}\right| w_{l}\right\rangle$. To improve the convergence rate of the expansion, we perform an autoregressive extrapolation [69] and we quench the Gibbs oscillations with the Jackson kernel [70].

\footnotetext{
${ }^{1}$ The MPS-KPM algorithm can be performed with a Hamiltonian whose full-spectrum is scaled and shifted to fit the interval $(-1,1)$, which for computational efficiency should be done in with the spectral center located at 0 .
}

\section{DYNAMICAL EXCITATIONS IN AN INTERACTING TOPOLOGICAL SUPERCONDUCTOR}

\section{A. Zero modes in interacting topological superconductors}

In this section, we first show how the previous formalism allows capturing the robustness of Majorana zero modes [71], a well-studied topological state that emerges taking $n=2$ in the generalized parafermion model. To go beyond the single-particle Majorana limit, we will benchmark our tensor network formalism with an interacting topological superconductor. In particular, it is well known that the ground state degeneracy of a finite island is not lifted by many-body interactions $[72,73]$ and that the zero-bias peak structure survives [74]. We take the following many-body Hamiltonian for an interacting for a one-dimensional topological superconductor:

$$
\begin{aligned}
\mathcal{H}= & \mu \sum_{n} c_{n}^{\dagger} c_{n}+t \sum_{n} c_{n}^{\dagger} c_{n+1}+\Delta \sum_{n} c_{n} c_{n+1} \\
& +V \sum_{n}\left(c_{n}^{\dagger} c_{n}-\frac{1}{2}\right)\left(c_{n+1}^{\dagger} c_{n+1}-\frac{1}{2}\right)+\text { H.c. }
\end{aligned}
$$

where $c_{n}^{\dagger}, c_{n}$ and the creation and annihilation fermionic operators, $\mu$ is the chemical potential, $t$ the hopping, $\Delta$ the $p$-wave superconducting order, and $V$ the electron-electron interaction. In the case of $V=0$, the previous Hamiltonian corresponds to a noninteracting one dimensional topological superconductor, whose eigenstates can be solved with a conventional Bogoliubov-de Gennes transformation [71]. This limit of $V=0$ corresponds to the Hamiltonian of Eq. (6) when taking $Z_{2}$ operators. In this limit $V=0$, the previous Hamiltonian of Eq. (12) is known to show edge zero modes. In particular, those zero modes are associated with Majorana excitation, one in each edge of the chain, that together encode a net twofold degeneracy of the ground state. In the noninteracting regime of $V=0$, these zero modes can be understood as arising from a nontrivial topological invariant of the associated Bogoliuvov-de-Gennes Hamiltonian $[7,71,75]$.

In the presence of interactions $V \neq 0$, the conventional single-particle classification no longer holds, and the Hamiltonian becomes purely many body. However, it is known that interactions do not lift the twofold degeneracy of an open Majorana chain $[72,73]$. The existence of twofold degeneracy is associated with the emergence of a zero-energy peak at the edge coexisting with a gapped bulk spectra in the spectral function

$$
A(\omega, n)=\left\langle\mathrm{GS}\left|c_{n} \delta\left(\omega-\mathcal{H}+E_{\mathrm{GS}}\right) c_{n}^{\dagger}\right| \mathrm{GS}\right\rangle,
$$

where $E_{\mathrm{GS}}$ is the ground state energy. This can be observed by computing the dynamical correlator of Eq. (13) at the edge and the bulk of the sample as the interaction $V$ is turned on Figs. 2(a) and 2(b). In particular, for $V=0$ the gapped bulk and zero-energy peak can be understood from the singleparticle picture as mentioned above. As the interaction $V$ is increased, a finite gap remains in bulk [Fig. 2(a)], and the zeroenergy peak remains [Fig. 2(b)]. At large enough interaction strengths, the bulk gap would close, and the zero-energy peak would get mixed the bulk states. The previous phenomenology shows that, as long as interactions are not strong enough to close the bulk gap, the Majorana zero-energy edge mode is 

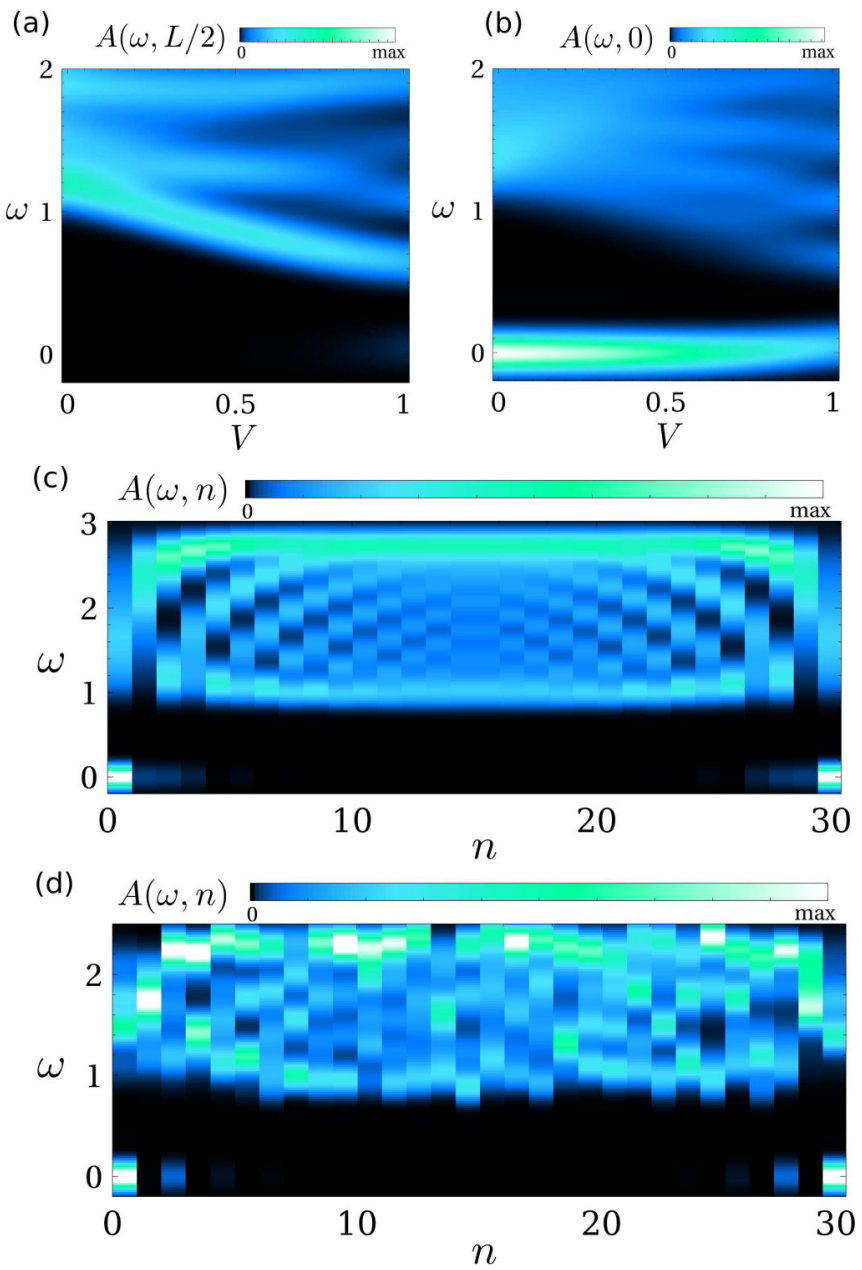

FIG. 2. Spectral function in the bulk (a) and at the edge (b) of a topological superconductor as a function of the interaction strength $V$. (c) shows the spectral function in the different sites of the chain for the interacting topological superconductor, showing the presence of zero-energy modes. (d) shows the spectral function in the different sites of the chain including both interactions and disorder, highlighting the robustness of the zero-energy excitations.

robust. This can also be observed by computing the spectral function of Eq. (13) in the different sites of the chain at an intermediate interaction $V$ as shown in Fig. 2(c). In particular, it is clearly observed that the zero-energy modes are strongly located at the edge and that they rapidly decay inside the chain, leading to a gapped bulk spectra [Fig. 2(c)].

In the discussion above, we have considered an interacting Hamiltonian, whose terms are uniform in space. It is, however, worth to note that these topological zero-energy modes remain robust in the presence of disorder in the Hamiltonian, both in the noninteracting and in the interacting regime. This can be explicitly shown by adding a disorder term to the Hamiltonian of Eq. (12) of the form $\mathcal{H}_{d}=\sum_{n} \epsilon_{n} c_{n}^{\dagger} c_{n}$ where $\epsilon_{n}$ is a different random number for each site in the interval $(-\epsilon, \epsilon)$. As shown in Fig. 2(d), the edge zero modes survive in the presence of this random disorder and interactions, whereas the gapped bulk states are heavily affected by it. This resilience of the zero modes is associated with their topological nature, sig- naling that for a moderate disorder strengths the topological degeneracy of the ground state remains invariant.

\section{B. Interface excitations in coupled topological superconductors}

Previously we showed that the topological zero modes appear at the edge of the one-dimensional chain, both in the presence of electronic interactions and disorder. We will now address how these topological zero modes would emerge a single chain is decoupled into two, which will lead to edge modes at each end of each subsystem. For this goal, we now define a parametric Hamiltonian, in which the coupling between the left and right parts is controlled by $\lambda$.

$$
\begin{aligned}
\mathcal{H}_{\lambda}= & \mu \sum_{n} c_{n}^{\dagger} c_{n}+t \sum_{n \neq L / 2} c_{n}^{\dagger} c_{n+1}+\Delta \sum_{n \neq L / 2} c_{n} c_{n+1} \\
& +V \sum_{n \neq L / 2}\left(c_{n}^{\dagger} c_{n}-\frac{1}{2}\right)\left(c_{n+1}^{\dagger} c_{n+1}-\frac{1}{2}\right) \\
& +\lambda\left[t c_{L / 2}^{\dagger} c_{L / 2+1}+\Delta c_{L / 2} c_{L / 2+1}\right] \\
& +\lambda\left[V\left(c_{L / 2}^{\dagger} c_{L / 2}-\frac{1}{2}\right)\left(c_{L / 2+1}^{\dagger} c_{L / 2+1}-\frac{1}{2}\right)\right] \\
& + \text { H.c. }
\end{aligned}
$$

By definition, $\lambda=1$ corresponds to the pristine limit of Eq. (12), whereas $\lambda=0$ corresponds to the fully decoupled limit in which the system consists of two independent chains. In this limit, the Hamiltonian consists of two fully decoupled chains, and therefore each chain develops its own pair of Majorana edge modes. The evolution from the fully coupled to the fully decoupled limit can be systematically explored by computing the the spectral function in the chain for different strengths of the coupling $\lambda$ as shown in Figs. 3(a)-3(d). As it is obvious to expect, coupling the two chains will lift away the interface zero modes. In particular, as the chains are decoupled, an interface state emerges and drifts to lower energies [Figs. 3(a)-3(d)]. This can be systematically studied by looking at the evolution of the spectral function at the interface as a function of the coupling $\lambda$, as shown in Fig. 3(e). In particular, the zero mode in the fully decoupled regime becomes a finite energy excitation as the coupling between the two chains is increased. Similar phenomenology is known in noninteracting Majorana chains, highlighting that the emergence of finite energy excitations from coupled topological zero modes also holds in the purely many-body regime. In the following, we will show that an analogous phenomenology happens in interacting parafermion chains.

\section{ZERO-MODE EXCITATIONS IN PARAFERMION CHAINS}

We now move on to consider chains of $Z_{3}$ parafermions, in particular building on top of the previous results for an interacting topological superconductor. The first interesting issue to consider is the many-body degeneracy of the parafermion chain, in comparison with the one of the topological superconductor [22]. This can be observed by analyzing the excitation energies as a function of the system size, as shown in Fig. 4(a). It is observed that as the system size becomes bigger, the 

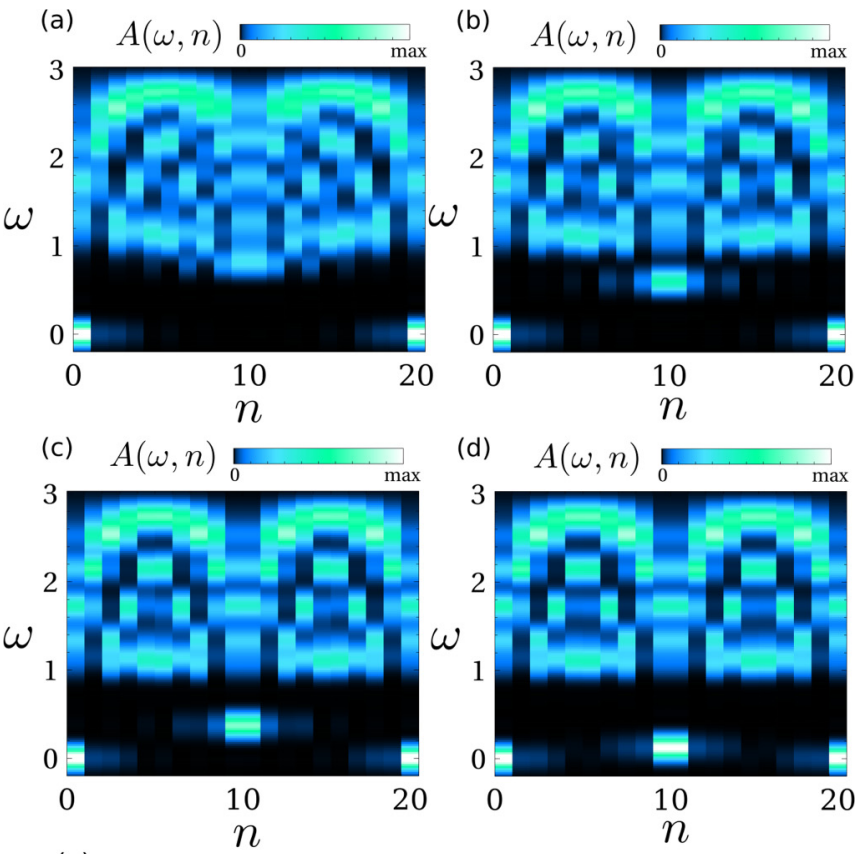

(e)

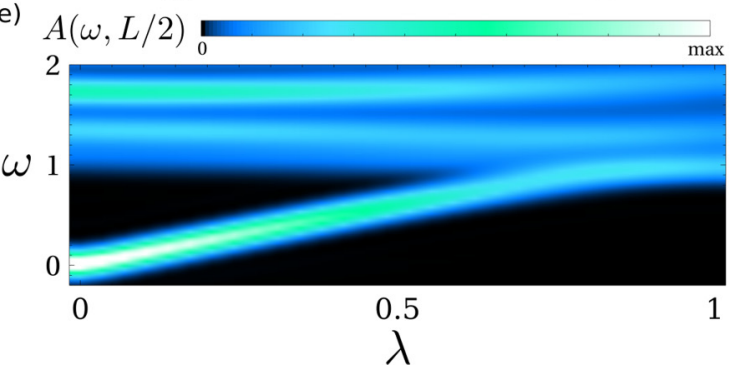

FIG. 3. [(a)-(d)] Spectral function of Eq. (13) in an interacting topological superconductor as a function of the coupling $\lambda$ between the left and right parts, with (a) $\lambda=0.7$, (b) 0.5 , (c) 0.3 , and (d) 0.1 . As the coupling $\lambda$ becomes weaker, interface modes move to lower energies, eventually giving rise to zero-energy excitations in the decoupled chain. (e) shows the spectral function at the edge as a function of the decoupling, highlighting the emergence of the interfacial zero mode at $\lambda=0$. We took $t=1, \Delta=0.6, \mu=-0.3$, and $V=0.3$ in (a)-(e).

energies of the first two excited states become arbitrarily close to the ground state energy, with the next excited state presenting a finite gap. This very same phenomenology takes place for the Majorana model, in which the finite splitting of the states for small chains is rationalized in terms of the hybridization between the edge modes. It is important to note that, in contrast with the Majorana model, the ground state of the $Z_{3}$ parafermion chain becomes threefold degenerate, in comparison with the twofold degeneracy of the Majorana chain.

In the case of a topological superconductor, the degeneracy of the ground state is associated with the emergence of Majorana zero modes at the edges. The degeneracy of the ground state with open boundary conditions for the $Z_{3}$ parafermion chain is again rationalized in terms of emergent topological edge modes, but now encoding a threefold degeneracy. This can be observed in the dynamical correlator computed at the edge of a parafermion chain as a function of the chain length, (a)

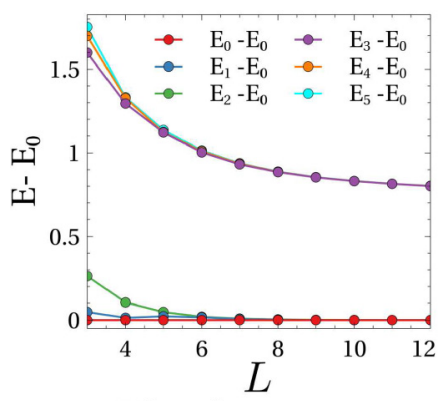

(b)
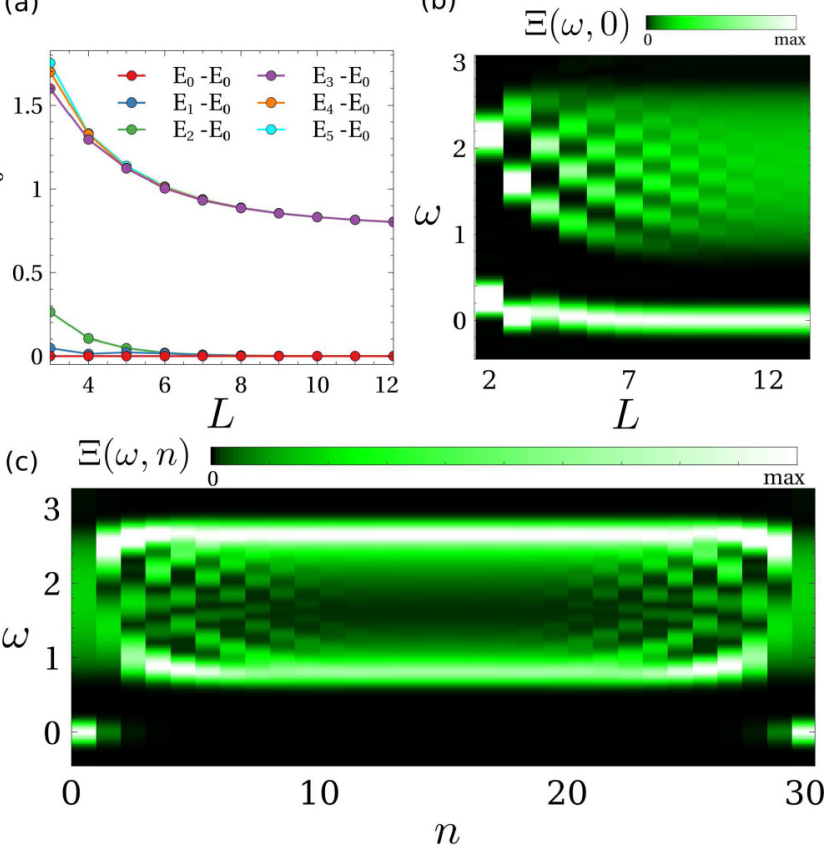

FIG. 4. (a) Many-body excitation energies of a parafermion chain with open boundary conditions, showing the emergence of a threefold degenerate ground state in the thermodynamic limit. (b) shows the spectral function at the edge as a function of the system size. (c) shows the spatially resolved spectral function, showing the emergence of edge excitations.

as showed in Fig. 4(b). In this fashion, the finite splitting between the lowest three energy levels for small chains can be rationalized in terms of a finite hybridization between the topological zero modes located at opposite edges. Due to the existence of a finite gap in the bulk of the chain, the zero modes are exponentially localized, leading to an exponential dependence of the hybridization between the states. This can be verified by looking at the spectral function for every site in the parafermion chain, as shown in Fig. 4(c). In particular, the topological zero modes are strongly localized at edges of the chain, whereas the spectral function remains gapped in the bulk of the chain [Fig. 4(c)]. In the next section, we will address the robustness of the edge zero-mode excitations, showing that the previous phenomenology is robust towards perturbations.

\section{PERTURBATIONS AND DISORDER IN PARAFERMION CHAINS}

Previously we focused on the pristine parafermionic chain showing the emergence of topological excitations at zero energy at the edge. In the following, we will assess the robustness of previous zero modes with respect to perturbations. In particular, we will focus on two different interaction terms, a biquadratic interaction between parafermions, and a next to nearest-neighbor hopping in the parafermion chain. We will examine the impact of these perturbations by computing the edge and bulk spectral function as the interaction term is increased, as it was shown in the interacting topological superconductor above. 

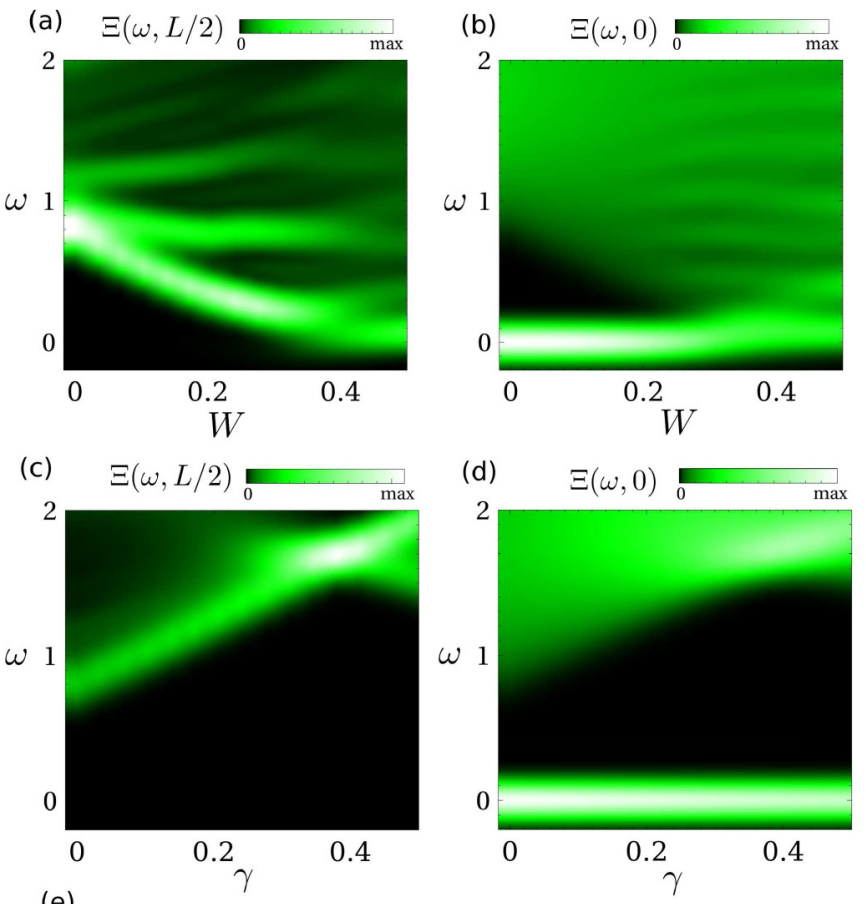

(e)

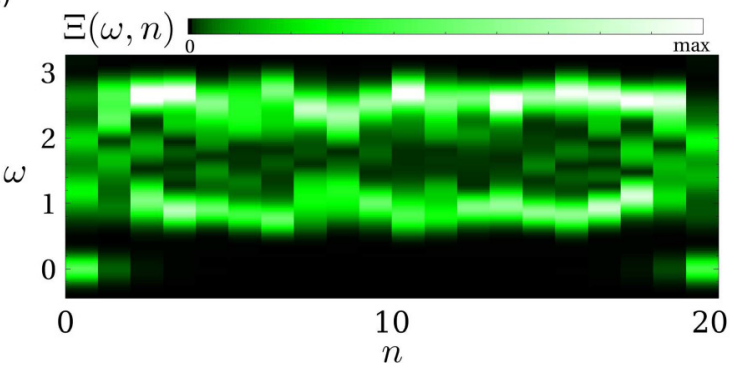

FIG. 5. [(a) and (b)] Spectral function in the bulk (a) and at the edge (b) as a function of the biquadratic interaction term of Eq. (15). [(c) and (d)] Spectral function in the bulk (c) and at the edge (d) as a function of the second-neighbor hopping of Eq. (16). (e) shows the spectral function in every site for the parafermion chain with random disorder of Eq. (17).

Let us first address the case of biquadratic interactions. In particular, we now include a term in the Hamiltonian that involves four parafermionic operators, leading to a Hamiltonian of the form

$$
\begin{aligned}
\mathcal{H}_{W}= & i \sum_{n} f \chi_{n}^{\dagger} \psi_{n}+i \theta \sum_{n} \psi_{n}^{\dagger} \chi_{n+1} \\
& +W \sum_{n} \psi_{n}^{\dagger} \chi_{n} \psi_{n+1}^{\dagger} \chi_{n+1}+\text { H.c. }
\end{aligned}
$$

where $W$ controls the strength of the biquadratic interaction. We compute the spectral function in bulk and at the edge as a function of the coupling parameter $W$, as shown in Figs. 5(a) and 5(b). In particular, we observe that as the interaction term is ramped up, the bulk spectral gap decreases. However, as long as the bulk gap remains open, the topological edge excitation remained pinned at zero energy. This phenomenology emphasizes that the biquadratic interaction parametrized by $W$ competes with the topological gap. However, as long as such perturbation is not strong enough to close the bulk gap,
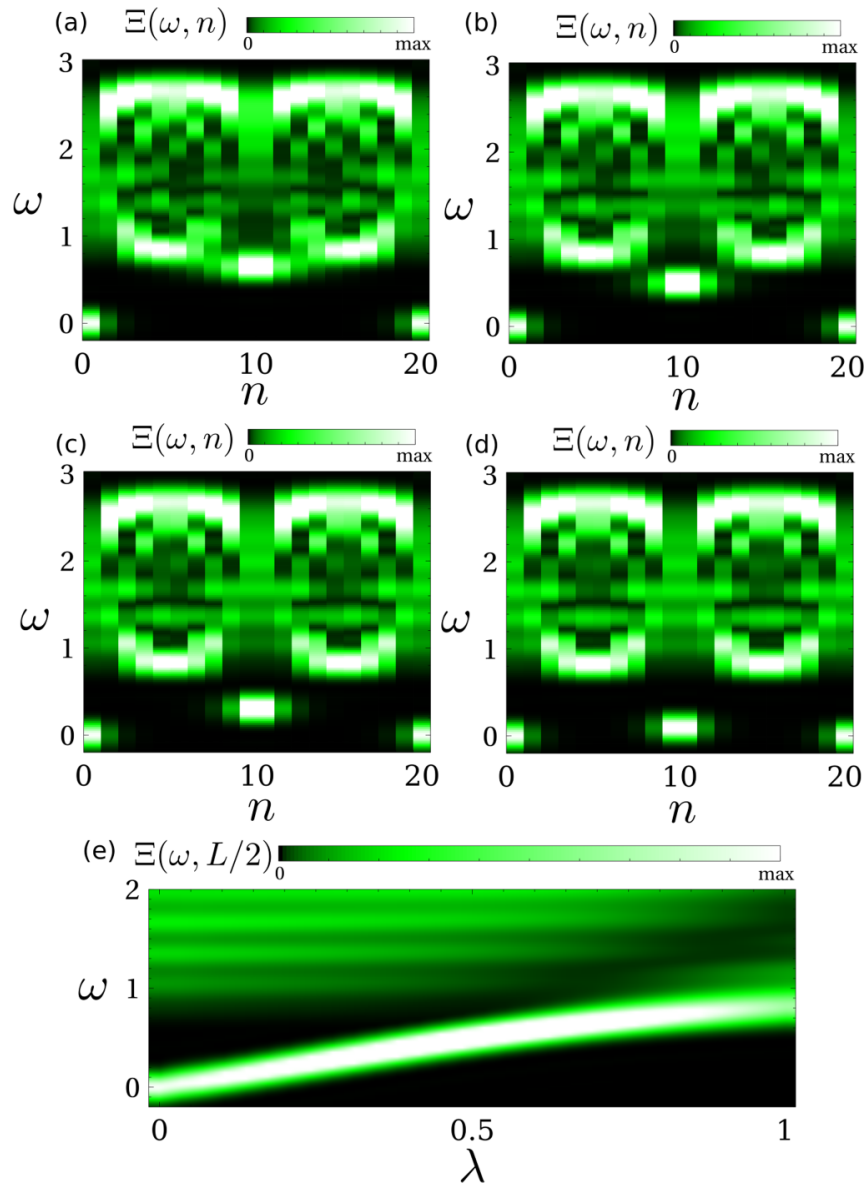

FIG. 6. [(a)-(d)] Spectral function in the different sites of two coupled parafermion chains, for different values of the interface coupling $\lambda$ between the left and right parts. We took (a) $\lambda=0.7$, (b) 0.5 , (c) 0.3 , and (d) 0.1 . As the coupling $\lambda$ is decreased, interface modes move towards lower energies, eventually giving rise to topological modes in the two decoupled chains. (e) shows the spectral function at the interface as a function of the coupling, highlighting the emergence of the interfacial zero mode at $\lambda=0$.

the topological edge excitations will remain pinned at zero energy. From the point of view of the degeneracy of the ground state of the parafermion chain, this means that a threefold degeneracy is robust against the biquadratic perturbation. It is interesting to note that this is an analogous phenomenology as the one shown above for the Majorana chain.

After showing that first neighbor interactions compete with the topological phase, we now turn to a different perturbation whose effect is dramatically different. We now consider a bilinear term in the parafermion Hamiltonian, giving rise to a second neighbor hopping. The full Hamiltonian now becomes

$$
\mathcal{H}_{\gamma}=i \sum_{n} f \chi_{n}^{\dagger} \psi_{n}+i \theta \sum_{n} \psi_{n}^{\dagger} \chi_{n+1}+\gamma \sum_{n} \psi_{n}^{\dagger} \chi_{n+2}+\text { H.c. }
$$

where $\gamma$ parametrizes the strength of a second-neighbor hopping between the parafermion operators. We show the spectral function in the bulk as a function of the coupling parameter $\lambda$ in Fig. 6. In particular, we see that the spectral function in the bulk increases its gap as $\gamma$ is ramped up. At 
the same time, the edge spectral function keeps showing a zero-energy resonance corresponding to the topological edge state. This phenomenology highlights that perturbations to the parafermionic Hamiltonian can also enhance the topological gap, and more importantly keeping the excitations pinned at zero energy at the edge.

It is finally interesting to show that the emergence of zero modes is not associated with the translational symmetry of the lattice. In particular, we now consider a Hamiltonian parafermion lattice where the couplings are disordered

$$
\mathcal{H}_{\mathrm{dis}}=i \sum_{n} f_{n} \chi_{n}^{\dagger} \psi_{n}+i \theta \sum_{n} \psi_{n}^{\dagger} \chi_{n+1}+\text { H.c. }
$$

where now $f_{n}$ takes random values between $(0.37,0.62)$. We compute the spatially resolved spectral function as shown in Fig. 5(e). It is seen that the edge zero modes survive in this disordered chain, despite the strong effect on the bulk states. This phenomenology demonstrates the robustness of the zeroenergy modes of the parafermion chains, and in particular, their existence is not associated with lattice symmetries. The next section addresses how coupling different topological modes between different chains lifts those excitations from zero energy.

\section{INTERFACE EXCITATIONS IN COUPLED PARAFERMION CHAINS}

Previously, we showed that weak perturbations to the parafermion Hamiltonian to not lift the edge excitations from zero energy. We now explore how topological excitations at zero energy can be created by weakly coupling two parafermion chains. For this sake, we define a parametric Hamiltonian of the form

$$
\mathcal{H}_{\mathrm{dis}}=i f \sum_{n} \chi_{n}^{\dagger} \psi_{n}+i \theta \sum_{n \neq L / 2} \psi_{n}^{\dagger} \chi_{n+1}+i \lambda \theta \psi_{L / 2}^{\dagger} \chi_{L / 2+1}+\text { H.c., }
$$

where $\lambda$ controls the coupling between two halves of the chain. In particular, for $\lambda=1$ the system corresponds to a uniform chain, whereas for $\lambda=0$, the system is formed for two decoupled chains.

Let us now look at the spectral function at every site as a function of the coupling strength between the two chains $\lambda$, as shown in Figs. 6(a)-6(d). Like in the Majorana case, it is is obvious to expect that coupling the two chains will lift away the interface zero-energy excitations. In the pristine case, $\lambda=$ 1 zero-edge excitations emerge at the two edges, coexisting with a fully gapped bulk. Starting with a finite but not perfect coupling $\lambda=0.7$ [Fig. 6(a)], we observe that a finite energy excitation starts to appear at the interface between the two chains. As the coupling between the two halves in weakened, an in-gap state drifts towards lower energies [Figs. 6(b)-6(d)], ultimately creating zero modes at the edges of the now two decoupled chains. This can also be systematically explored by computing the spectral function at the interface between the two chains as a function of $\lambda$, as shown in Fig. 6(e). It is clearly observed that the two topological edge modes, originally located at zero energy, become finite energy excitations as the coupling between the two chains is increased. This shares the same phenomenology as conventional Majorana chains, highlighting that the hybridization between topological zero modes generically give rise to finite energy excitations. It is finally interesting to note that for $\lambda \neq 0$, the collective ground state of the two chains will be threefold degenerate in the thermodynamic limit. In contrast, for $\lambda=0$ the ground state becomes ninefold degenerate. For $\lambda \neq 0$, the first excited state will then correspond to the interface excitation that arises from the coupled edge modes at the junction, whose energy can be inferred from the spectral function of Fig. 6(e). These results highlight that coupling topological excitations is an effective way of creating in-gap modes at finite energy.

\section{CONCLUSIONS}

To summarize, we have shown the emergence of zero modes and excitations at finite energies in a parafermion chain. Generic parafermion models are challenging to study analytically, and their topological excitations are less understood than those of single-particle topological systems. To study this interacting model, we employed a combination of tensor network and kernel polynomial techniques that allow addressing the full excitation spectra of the interacting Hamiltonian. We have shown that topological parafermion chains feature robust zero-energy excitations, that encode the threefold degeneracy of the ground state in the thermodynamic limit. We demonstrated that these excitations are robust against perturbations, including biquadratic interactions, second neighbor hopping and disorder. We then showed how interfacial modes at finite energies can be created by weakly coupling different parafermion chains, with an excitation energy controllable by the coupling between the chains. Our results demonstrate the robustness of these topological excitations in parafermion chains, and put forward kernel polynomial tensor networks as a versatile technique to study finite-energy excitations in highly interacting models.

\section{ACKNOWLEDGMENTS}

We acknowledge the computational resources provided by the Aalto Science-IT project. J.L.L. is grateful for financial support from the Academy of Finland Projects No. 331342 and No. 336243.

\section{APPENDIX: RELATION BETWEEN $A(\omega, n)$ AND THE SINGLE-PARTICLE EXCITATION ENERGIES}

Here we show that, in the noninteracting limit, the spectral function of Eq. (13) corresponds to the single-particle density of states.

Let us take a single particle Hamiltonian of the form $\mathcal{H}=\sum_{i j} H_{i j} c_{i}^{\dagger} c_{j}$. In its diagonal form, it becomes $\mathcal{H}=$ $\sum_{k} \epsilon_{k} d_{k}^{\dagger} d_{k}$, where $d_{k}$ are the single particle eigenstates and $\epsilon_{k}$ the single particle eigenenergies. A unitary transformation $U$ relates the $c_{n}^{\dagger}$ and $d_{k}^{\dagger}$ operators as $c_{n}^{\dagger}=\sum_{k} U_{k, n} d_{k}^{\dagger}$. We start with the single-particle formalism to compute the local spectral function, also known as the local density of states. For the single-particle formalism, we take a basis of single-electron states $|n\rangle=c_{n}^{\dagger}|0\rangle$, where $|0\rangle$ is the vacuum state. In this case, we would compute the local density of states as $D(\omega, n)=\langle n|\delta(\omega-H)| n\rangle \sim\left\langle n\left|\operatorname{Im}\left[(\omega-H)^{-1}\right]\right| n\right\rangle$, 
where $H$ is the tight-binding matrix. In the diagonal basis, it takes the form $D(\omega, n)=\sum_{k}\left|U_{k, n}\right|^{2} \delta\left(\omega-\epsilon_{k}\right)$, which is the conventional form for the local spectral function.

We now move on to the computation in the spectral function working in the many-body Fock space as done in DMRG [76-79]. In this space, the basis is no-longer states with a single electron, but with an arbitrary number of electrons. The many-body ground state is given by the Fermi sea $|\mathrm{GS}\rangle=\Pi_{\epsilon_{k}<0} d_{k}^{\dagger}|0\rangle$, where $|0\rangle$ is the vacuum state $d_{k}|0\rangle=$ 0 . This state is, by definition, the eigenstate with the lowest possible energy associated to the Hamiltonian $\mathcal{H}$, with $\mathcal{H}|\mathrm{GS}\rangle=E_{\mathrm{GS}}|\mathrm{GS}\rangle$, and it has a total energy $E_{\mathrm{GS}}=\sum_{\epsilon_{k}<0} \epsilon_{k}$. The excited states can be build analogously. In particular, for each single-particle energy $\epsilon_{k^{\prime}}>0$, there is an excited manybody state with one more electron than the ground state given by $\left|k^{\prime}\right\rangle=d_{k^{\prime}}^{\dagger}|\mathrm{GS}\rangle$, with an energy $\mathcal{H}\left|k^{\prime}\right\rangle=\left(E_{\mathrm{GS}}+\epsilon_{k^{\prime}}\right)\left|k^{\prime}\right\rangle$. With the previous points, let us now move on to consider the dynamical correlator of Eq. (13) $A(\omega, n)=\langle\mathrm{GS}| c_{n} \delta(\omega-$
$\left.\mathcal{H}+E_{\mathrm{GS}}\right) c_{n}^{\dagger}|\mathrm{GS}\rangle$. In the Fock space, the term $\delta(\omega-H+$ $\left.E_{\mathrm{GS}}\right)$ takes the form $\sum_{\Psi} \delta\left(\omega-E_{\Psi}+E_{\mathrm{GS}}\right)|\Psi\rangle\langle\Psi|$, where $E_{\Psi}$ is the many-body energy of the many-body eigenstate $|\Psi\rangle$. With the previous representation and taking the definitions of the different terms, we get $A(\omega, n)=\sum_{\epsilon_{k}>0}\left|U_{k, n}\right|^{2} \delta\left(\omega-\epsilon_{k}\right)$, the conventional definition of the local density of states for a single particle Hamiltonian. In other words, the function $A(\omega, n)$ directly reflects the single particle energies $\epsilon_{k}>0$, namely the unoccupied single particle states. As a result, the spectral function computed with Eq. (13) corresponds to the conventional single-particle spectral function as $D(\omega>$ $0, n)=A(\omega, n)$. We note that the full local density of states can be computed analogously as $D(\omega, n)=\langle\mathrm{GS}| c_{n} \delta(\omega-$ $\left.\mathcal{H}+E_{\mathrm{GS}}\right) c_{n}^{\dagger}|\mathrm{GS}\rangle+\left\langle\mathrm{GS}\left|c_{n}^{\dagger} \delta\left(-\omega-\mathcal{H}+E_{\mathrm{GS}}\right) c_{n}\right| \mathrm{GS}\right\rangle$. For an interacting system that lacks a single particle description, the previous formalism allows computing the spectral function generically, and therefore it is the method used in our manuscript.
[1] M. Z. Hasan and C. L. Kane, Colloquium: Topological insulators, Rev. Mod. Phys. 82, 3045 (2010).

[2] X.-L. Qi and S.-C. Zhang, Topological insulators and superconductors, Rev. Mod. Phys. 83, 1057 (2011).

[3] A. P. Schnyder, S. Ryu, A. Furusaki, and A. W. W. Ludwig, Classification of topological insulators and superconductors in three spatial dimensions, Phys. Rev. B 78, 195125 (2008).

[4] C.-K. Chiu, J. C. Y. Teo, A. P. Schnyder, and S. Ryu, Classification of topological quantum matter with symmetries, Rev. Mod. Phys. 88, 035005 (2016).

[5] C. L. Kane and E. J. Mele, Quantum Spin Hall Effect in Graphene, Phys. Rev. Lett. 95, 226801 (2005).

[6] M. Sato and Y. Ando, Topological superconductors: A review, Rep. Prog. Phys. 80, 076501 (2017).

[7] C.W.J. Beenakker, Search for majorana fermions in superconductors, Annu. Rev. Condens. Matter Phys. 4, 113 (2013).

[8] E. Majorana, Teoria simmetrica dell'elettrone e del positrone, Il Nuovo Cimento 14, 171 (1937).

[9] C. Nayak, S. H. Simon, A. Stern, M. Freedman, and S. Das Sarma, Non-Abelian anyons and topological quantum computation, Rev. Mod. Phys. 80, 1083 (2008).

[10] J. Alicea, Y. Oreg, G. Refael, F. von Oppen, and M. P. A. Fisher, Non-abelian statistics and topological quantum information processing in 1d wire networks, Nat. Phys. 7, 412 (2011).

[11] D. Aasen, M. Hell, R. V. Mishmash, A. Higginbotham, J. Danon, M. Leijnse, T. S. Jespersen, J. A. Folk, C. M. Marcus, K. Flensberg, and J. Alicea, Milestones Toward Majorana-Based Quantum Computing, Phys. Rev. X 6, 031016 (2016).

[12] R. M. Lutchyn, T. D. Stanescu, and S. Das Sarma, Search for Majorana Fermions in Multiband Semiconducting Nanowires, Phys. Rev. Lett. 106, 127001 (2011).

[13] Y. Oreg, G. Refael, and F. von Oppen, Helical Liquids and Majorana Bound States in Quantum Wires, Phys. Rev. Lett. 105, 177002 (2010).

[14] T. D. Stanescu, R. M. Lutchyn, and S. Das Sarma, Majorana fermions in semiconductor nanowires, Phys. Rev. B 84, 144522 (2011).
[15] J. Röntynen and T. Ojanen, Topological Superconductivity and High Chern Numbers in 2D Ferromagnetic Shiba Lattices, Phys. Rev. Lett. 114, 236803 (2015).

[16] P. San-Jose, J. L. Lado, R. Aguado, F. Guinea, and J. FernándezRossier, Majorana Zero Modes in Graphene, Phys. Rev. X 5, 041042 (2015).

[17] J. Klinovaja, P. Stano, A. Yazdani, and D. Loss, Topological Superconductivity and Majorana Fermions in Rkky Systems, Phys. Rev. Lett. 111, 186805 (2013).

[18] J. D. Sau, R. M. Lutchyn, S. Tewari, and S. Das Sarma, Generic New Platform for Topological Quantum Computation Using Semiconductor Heterostructures, Phys. Rev. Lett. 104, 040502 (2010).

[19] J. L. Lado and M. Sigrist, Two-Dimensional Topological Superconductivity with Antiferromagnetic Insulators, Phys. Rev. Lett. 121, 037002 (2018).

[20] L. Fu and C. L. Kane, Superconducting Proximity Effect and Majorana Fermions at the Surface of a Topological Insulator, Phys. Rev. Lett. 100, 096407 (2008).

[21] L. Jiang, T. Kitagawa, J. Alicea, A. R. Akhmerov, D. Pekker, G. Refael, J. I. Cirac, E. Demler, M. D. Lukin, and P. Zoller, Majorana Fermions in Equilibrium and in Driven Cold-Atom Quantum Wires, Phys. Rev. Lett. 106, 220402 (2011).

[22] P. Fendley, Parafermionic edge zero modes in Zn-invariant spin chains, J. Stat. Mech.: Theory Exp. (2012) P11020.

[23] A.Yu. Kitaev, Fault-tolerant quantum computation by anyons, Ann. Phys. 303, 2 (2003).

[24] J. K. Jain, Composite-Fermion Approach for the Fractional Quantum Hall Effect, Phys. Rev. Lett. 63, 199 (1989).

[25] N. Read and E. Rezayi, Beyond paired quantum hall states: Parafermions and incompressible states in the first excited landau level, Phys. Rev. B 59, 8084 (1999).

[26] N. Schiller, E. Cornfeld, E. Berg, and Y. Oreg, Predicted signatures of topological superconductivity and parafermion zero modes in fractional quantum hall edges, Phys. Rev. Res. 2, 023296 (2020).

[27] W. Zhu, S. S. Gong, F. D. M. Haldane, and D. N. Sheng, Fractional Quantum Hall States at $v=13 / 5$ and $12 / 5$ and 
their Non-Abelian Nature, Phys. Rev. Lett. 115, 126805 (2015).

[28] A. Vaezi, Superconducting Analog of the Parafermion Fractional Quantum Hall States, Phys. Rev. X 4, 031009 (2014).

[29] M. Barkeshli and X.-G. Wen, Effective field theory and projective construction for $Z_{k}$ parafermion fractional quantum hall states, Phys. Rev. B 81, 155302 (2010).

[30] A. C. Balram, M. Barkeshli, and M. S. Rudner, Parton construction of particle-hole-conjugate read-rezayi parafermion fractional quantum hall states and beyond, Phys. Rev. B 99, 241108(R) (2019).

[31] L. H. Santos, Parafermions in hierarchical fractional quantum hall states, Phys. Rev. Res. 2, 013232 (2020).

[32] M. Barkeshli and X.-G. Wen, $u(1) \times u(1) \rtimes Z_{2}$ chern-simons theory and $Z_{4}$ parafermion fractional quantum hall states, Phys. Rev. B 81, 045323 (2010).

[33] J. Behrmann, Z. Liu, and E. J. Bergholtz, Model Fractional Chern Insulators, Phys. Rev. Lett. 116, 216802 (2016).

[34] G. Moore and N. Read, Nonabelions in the fractional quantum hall effect, Nucl. Phys. B 360, 362 (1991).

[35] S. Groenendijk, A. Calzona, H. Tschirhart, E. G. Idrisov, and T. L. Schmidt, Parafermion braiding in fractional quantum hall edge states with a finite chemical potential, Phys. Rev. B 100, 205424 (2019).

[36] J. Alicea and P. Fendley, Topological phases with parafermions: Theory and blueprints, Annu. Rev. Condens. Matter Phys. 7, 119 (2016).

[37] L. Fidkowski and A. Kitaev, Topological phases of fermions in one dimension, Phys. Rev. B 83, 075103 (2011).

[38] J. H. Son and J. Alicea, Commuting-projector hamiltonians for chiral topological phases built from parafermions, Phys. Rev. B 97, 245144 (2018).

[39] M. A. Levin and X.-G. Wen, String-net condensation: A physical mechanism for topological phases, Phys. Rev. B 71, 045110 (2005).

[40] W. Li, S. Yang, H.-H. Tu, and M. Cheng, Criticality in translation-invariant parafermion chains, Phys. Rev. B 91, 115133 (2015).

[41] J. Klinovaja and D. Loss, Parafermions in an Interacting Nanowire Bundle, Phys. Rev. Lett. 112, 246403 (2014).

[42] A. M. Tsvelik, Integrable Model with Parafermion Zero Energy Modes, Phys. Rev. Lett. 113, 066401 (2014).

[43] A. S. Jermyn, R. S. K. Mong, J. Alicea, and P. Fendley, Stability of zero modes in parafermion chains, Phys. Rev. B 90, 165106 (2014).

[44] H. Ebisu, E. Sagi, Y. Tanaka, and Y. Oreg, Generalized parafermions and nonlocal josephson effect in multilayer systems, Phys. Rev. B 95, 075111 (2017).

[45] A. Alexandradinata, N. Regnault, C. Fang, M. J. Gilbert, and B. A. Bernevig, Parafermionic phases with symmetry breaking and topological order, Phys. Rev. B 94, 125103 (2016).

[46] N. Moran, D. Pellegrino, J. K. Slingerland, and G. Kells, Parafermionic clock models and quantum resonance, Phys. Rev. B 95, 235127 (2017).

[47] R. Samajdar, S. Choi, H. Pichler, M. D. Lukin, and S. Sachdev, Numerical study of the chiral $\mathbb{Z}_{3}$ quantum phase transition in one spatial dimension, Phys. Rev. A 98, 023614 (2018).

[48] A. Chew, D. F. Mross, and J. Alicea, Fermionized parafermions and symmetry-enriched majorana modes, Phys. Rev. B 98, 085143 (2018).
[49] D. Rossini, M. Carrega, M. Calvanese Strinati, and L. Mazza, Anyonic tight-binding models of parafermions and of fractionalized fermions, Phys. Rev. B 99, 085113 (2019).

[50] F. Iemini, C. Mora, and L. Mazza, Topological Phases of Parafermions: A Model with Exactly Solvable Ground States, Phys. Rev. Lett. 118, 170402 (2017).

[51] L. H. Santos and T. L. Hughes, Parafermionic Wires at the Interface of Chiral Topological States, Phys. Rev. Lett. 118, 136801 (2017).

[52] C. Chen and F. J. Burnell, Tunable Splitting of the Ground-State Degeneracy in Quasi-One-Dimensional Parafermion Systems, Phys. Rev. Lett. 116, 106405 (2016).

[53] G. Ortiz, E. Cobanera, and Z. Nussinov, Dualities and the phase diagram of the p-clock model, Nucl. Phys. B 854, 780 (2012).

[54] E. Fradkin and L. P. Kadanoff, Disorder variables and parafermions in two-dimensional statistical mechanics, Nucl. Phys. B 170, 1 (1980).

[55] Y. Zhuang, H. J. Changlani, N. M. Tubman, and T. L. Hughes, Phase diagram of the $Z_{3}$ parafermionic chain with chiral interactions, Phys. Rev. B 92, 035154 (2015).

[56] L. Mazza, J. Viti, M. Carrega, D. Rossini, and A. De Luca, Energy transport in an integrable parafermionic chain via generalized hydrodynamics, Phys. Rev. B 98, 075421 (2018).

[57] S. R. White, Density Matrix Formulation for Quantum Renormalization Groups, Phys. Rev. Lett. 69, 2863 (1992).

[58] S. R. White, Density-matrix algorithms for quantum renormalization groups, Phys. Rev. B 48, 10345 (1993).

[59] U. Schollwöck, The density-matrix renormalization group in the age of matrix product states, Ann. Phys. 326, 96 (2011).

[60] ITensor Library http://itensor.org.

[61] DMRGpy Library https://github.com/joselado/dmrgpy.

[62] M. Fishman, S. R. White, and E. M. Stoudenmire, The ITensor software library for tensor network calculations, arXiv:2007.14822.

[63] A. Weiße, G. Wellein, A. Alvermann, and H. Fehske, The kernel polynomial method, Rev. Mod. Phys. 78, 275 (2006).

[64] F. A. Wolf, J. A. Justiniano, I. P. McCulloch, and U. Schollwöck, Spectral functions and time evolution from the chebyshev recursion, Phys. Rev. B 91, 115144 (2015).

[65] H. D. Xie, R. Z. Huang, X. J. Han, X. Yan, H. H. Zhao, Z. Y. Xie, H. J. Liao, and T. Xiang, Reorthonormalization of chebyshev matrix product states for dynamical correlation functions, Phys. Rev. B 97, 075111 (2018).

[66] J. L. Lado and M. Sigrist, Solitonic in-gap modes in a superconductor-quantum antiferromagnet interface, Phys. Rev. Res. 2, 023347 (2020).

[67] J. L. Lado and O. Zilberberg, Topological spin excitations in harper-heisenberg spin chains, Phys. Rev. Res. 1, 033009 (2019).

[68] M. Rösner and J. L. Lado, Coulomb-engineered topology, arXiv:2008.07990.

[69] H. Akaike, Fitting autoregressive models for prediction, Ann. Inst. Stat. Math. 21, 243 (1969).

[70] D. Jackson, On approximation by trigonometric sums and polynomials, Trans. Am. Math. Soc. 13, 491 (1912).

[71] A. Yu. Kitaev, Unpaired majorana fermions in quantum wires, Phys. Usp. 44, 131 (2001). 
[72] A. M. Lobos, R. M. Lutchyn, and S. Das Sarma, Interplay of Disorder and Interaction in Majorana Quantum Wires, Phys. Rev. Lett. 109, 146403 (2012).

[73] E. M. Stoudenmire, J. Alicea, O. A. Starykh, and M. P. A. Fisher, Interaction effects in topological superconducting wires supporting majorana fermions, Phys. Rev. B 84, 014503 (2011).

[74] R. Thomale, S. Rachel, and P. Schmitteckert, Tunneling spectra simulation of interacting majorana wires, Phys. Rev. B 88, 161103(R) (2013).

[75] J. Alicea, New directions in the pursuit of majorana fermions in solid state systems, Rep. Prog. Phys. 75, 076501 (2012).
[76] R. Peters, Spectral functions for single- and multi-impurity models using density matrix renormalization group, Phys. Rev. B 84, 075139 (2011).

[77] A. Holzner, A. Weichselbaum, I. P. McCulloch, U. Schollwöck, and J. von Delft, Chebyshev matrix product state approach for spectral functions, Phys. Rev. B 83, 195115 (2011).

[78] K. A. Hallberg, Density-matrix algorithm for the calculation of dynamical properties of low-dimensional systems, Phys. Rev. B 52, R9827 (1995).

[79] P. E. Dargel, A. Honecker, R. Peters, R. M. Noack, and T. Pruschke, Adaptive lanczos-vector method for dynamic properties within the density matrix renormalization group, Phys. Rev. B 83, 161104(R) (2011). 